\title{
Epidemiology of dental root caries: a review of risk factors
}

\author{
Jingyang Zhang, Edward Chin Man Lo \\ Faculty of Dentistry, University of Hong Kong, 34 Hospital Road, Sai Ying Pun, Hong Kong, China \\ Contributions: (I) Conception and design: All authors; (II) Administrative support: None; (III) Provision of study materials or patients: None; (IV) \\ Collection and assembly of data: J Zhang; (V) Data analysis and interpretation: All authors; (VI) Manuscript writing: All authors; (VII) Final approval \\ of manuscript: All authors. \\ Correspondence to: Edward Chin Man Lo. Faculty of Dentistry, University of Hong Kong, 34 Hospital Road, Sai Ying Pun, Hong Kong, China. \\ Email: edward-lo@hku.hk.
}

Background and Objective: Root surface caries is a common disease among the older adults worldwide. This paper reports on two recent systematic reviews which were conducted to identify the risk factors of root caries, one on risk indicators and one on risk predictors, and to describe their relationship with the prevalence and incidence of root caries.

Methods: Articles in four electronic databases (PubMed, MEDLINE, EMBASE, and Scopus) published in 1990 to 2018 were searched. Epidemiological studies conducted on general populations and investigated at least one risk factor of root caries were included. Relevant information was extracted by two reviewers independently. The factors were put into five main categories, namely demographic and social background, health behaviors, general health, oral health habits and oral health conditions.

Key Content and Findings: Forty crosssectional surveys (78,183 participants in total) and 16 longitudinal cohort studies (7,340 participants in total) from different countries worldwide were included in the reviews. There were positive correlations between dental root caries and age, past root caries experience, exposure of root surfaces and use of tobacco. Negative correlations with socio-economic status and oral hygiene were found.

Conclusions: Higher risk of having or developing new root caries were found among the older adults, people with lower socio-economic status, tobacco users, and those with greater root caries experience, more gingival recession and more dental plaque.

Keywords: Dental caries; epidemiology; review; elderly

Received: 10 January 2020; Accepted: 28 February 2020; Published: 25 March 2020.

doi: $10.21037 /$ fomm.2020.03.02

View this article at: http://dx.doi.org/10.21037/fomm.2020.03.02

\section{Introduction}

Life expectancy and proportion of older adults in the population in many countries around the world has increased greatly in the recent decades (1). A recent review on the global burden of untreated caries found that both the prevalence and the incidence of dental caries increased after the age of 40 , and $35-40 \%$ of the older adults were affected (2). It is expected that a high proportion of the new caries in the older adults occurs in the roots of the teeth. For example, in the latest nation-wide oral health survey conducted in China, $62 \%$ of the adults aged $65-74$ years had root caries and they had a mean of 2.6 decayed and filled tooth roots (3). As people are retaining more teeth when they age, there is an anticipated increase in root caries over time. Dental root caries may lead to pain and loss of teeth, and even affect the general health status and affect the quality of life of the older adults. Therefore, management of this disease among the ageing adult population is an important issue in dental public health and there is a high demand for effective prevention programs (4).

Dental root caries is usually found on exposed root surface below or at the cemento-enamel junction (5). Diagnosis of root caries lesions is mainly by visual and 
tactile methods. Lesion location, texture (soft or penetrable upon gentle probing with a blunt probe), color (usually discolored), cavitation and contour of the root surfaces are all included for a holistic consideration (6). Root caries is caused by cariogenic bacteria fermenting carbohydrates on the exposed root surface. The tissues of tooth root are dentin and a thin layer of cementum. Compared to enamel covering the crown of tooth, dentin has much greater amount of organic component and lower mineral content. Demineralization of dentin occurs at a critical $\mathrm{pH}$ higher than that of enamel and the caries process involves degradation of collagen. Besides, the morphology of tooth crown and tooth root is very different. Thus, the risk factors and risk predictors for root caries may be different from those for coronal caries. Identifying the root caries risk factors will help to develop effective prevention methods and apply them to adults with higher caries risk.

\section{Methods}

The authors of this paper had recently conducted two systematic reviews of the published epidemiological studies on root caries, one on cross-sectional surveys and one on longitudinal cohort studies. Details of the two reviews and the findings were published in two articles $(7,8)$. The main findings of these two reviews are summarized and presented in this paper. The factors associated with root caries found in cross-sectional studies and the risk predictors identified in longitudinal studies are grouped into five categories: (I) socio-demographic; (II) general health status; (III) general health behaviors; (IV) oral health related practices; and (V) oral health conditions including clinical, microbiologic and salivary parameters.

Epidemiological studies conducted on general populations and investigated at least one risk factor of root caries were included in the systematic reviews. No language restriction was set in the search strategy. The PubMed, MEDLINE, EMBASE and Scopus databases were searched and papers published in 1990 to 2018 were included. Manual search was also carried out on the list of references in the selected articles and review papers. The exclusion criteria were: (I) studies without any statistical analysis of root caries data; (II) surveys with sample size smaller than 100; and (III) cohort studies with follow-up rate lower than $50 \%$. The Newcastle-Ottawa Scale, with necessary modification, was used in the assessment of the risk of bias of the included papers (9), and papers with low quality were removed. The final numbers of cross-sectional surveys and longitudinal cohort studies included in the two reviews were 40 and 16 , respectively.

Among the included cross-sectional surveys, most of them (27 out of 40) conducted random sampling in the community while some (8 out of 40) used convenience sampling. Both the prevalence and extent (the mean DFroot or D-root) of root caries were reported in 39 studies. While results regarding prevalence of root caries found in the crude (unadjusted bivariate analysis) or adjusted (statistical modelling accounting for confounding effects of other factors) data analysis of the studies were usually reported, most of the studies only reported the extent of root caries from the crude analysis. Nineteen studies reported both DFroot and D-root, while 3 and 18 studies reported DF-root (decayed and filled root) and D-root alone, respectively.

Among the 16 included cohort studies, three reported both the incidence and the increment of root caries, while six studies only reported on the incidence and seven studies only reported on the increment. In these studies, incidence and increment of root caries were analyzed through crude or adjusted data analysis. Seven studies only reported on DFroot, four studies only reported D-root while five studies reported on both DF-root and D-root. Regarding the root caries diagnostic criteria adopted in the reviewed studies, most of them reported that the examiners used visual-tactile examination but the exact criteria might not be specified.

\section{Socio-demographic factors}

\section{Age}

Among the 25 cross-sectional studies, 16 (64\%) of them found a positive correlation between age and root caries, and only one study found the contrary (Table 1). This finding is not surprising because dental caries develops slowly over time and DF-root score is an accumulative and irreversible measure. The age groups included in the surveys range from $65+$ years in the earlier time to as wide as $20-80$ years recently, showing a possible trend towards younger age for root caries. On the contrary, no consistent correlation between age and new root caries was found in the longitudinal studies (Table 2). In one study, the correlation between age and incidence of DF-root was positive while the correlation with incidence of D-root was negative (10). Among the other 8 out the 9 cohort studies, three studies reported a positive correlation while the other five studies did not find a significant relationship. Thus, although the older age groups usually have a higher 
Table 1 Association between selected factors and the prevalence or extent of decayed root or decayed and filled root

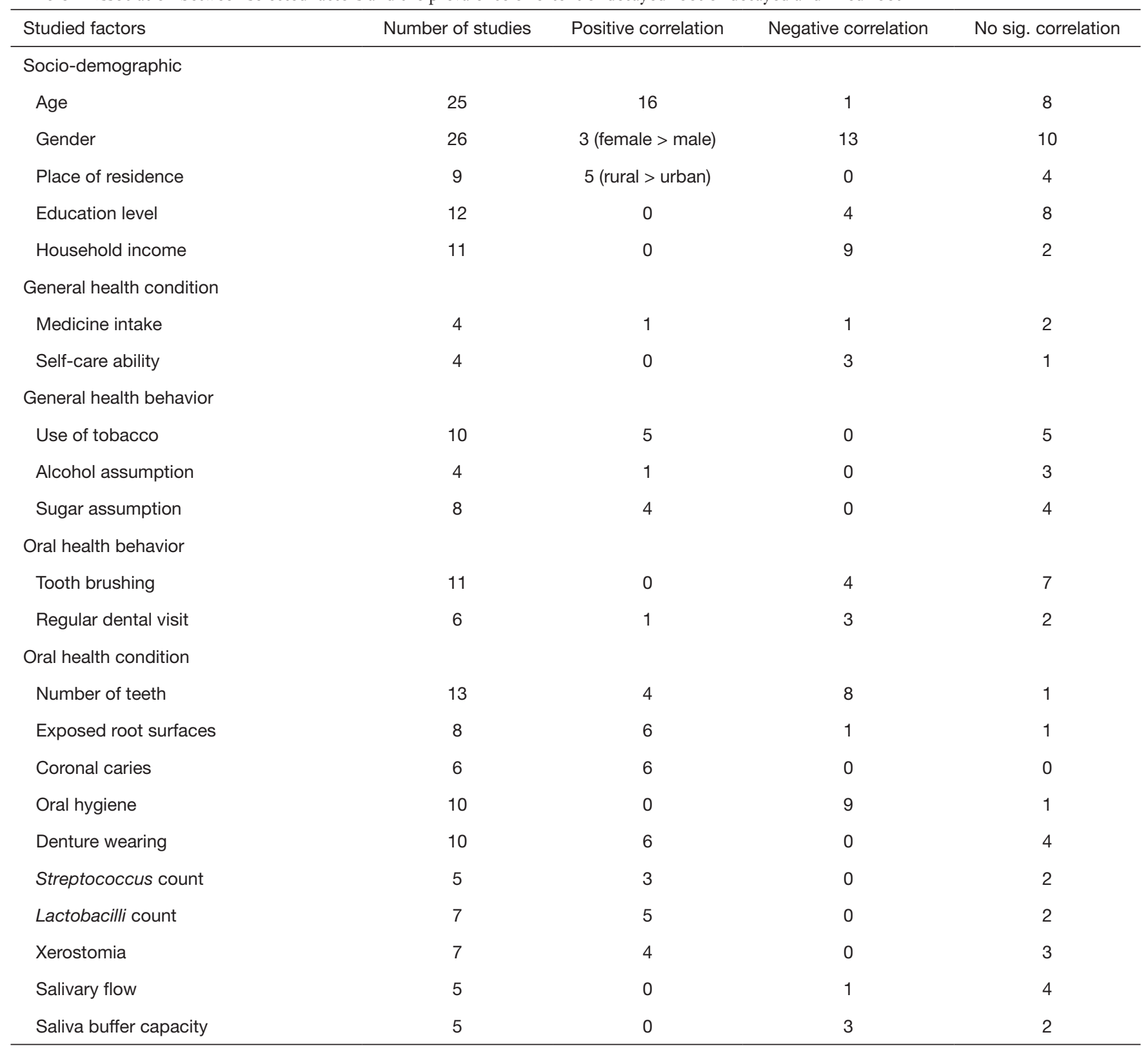

prevalence of root caries, age per se may not be a reliable predictor of new root caries.

\section{Gender}

Out of the 26 cross-sectional studies that had investigated the association between gender and root caries, 13 (50\%) studies found a higher prevalence among men, three studies found the opposite and ten studies reported no statistically significant correlation (Table 1). Among the seven cohort studies that investigated this aspect, $6(86 \%)$ did not find a statistically significant correlation between gender and incidence of root caries (Table 2). So, no clear conclusion on the relationship between gender and root caries can be drawn.

\section{Area of residence}

Slightly more than half (5 out of 9) of the included cross- 
Table 2 Association between selected factors and the incidence or increment of decayed root or decayed and filled root

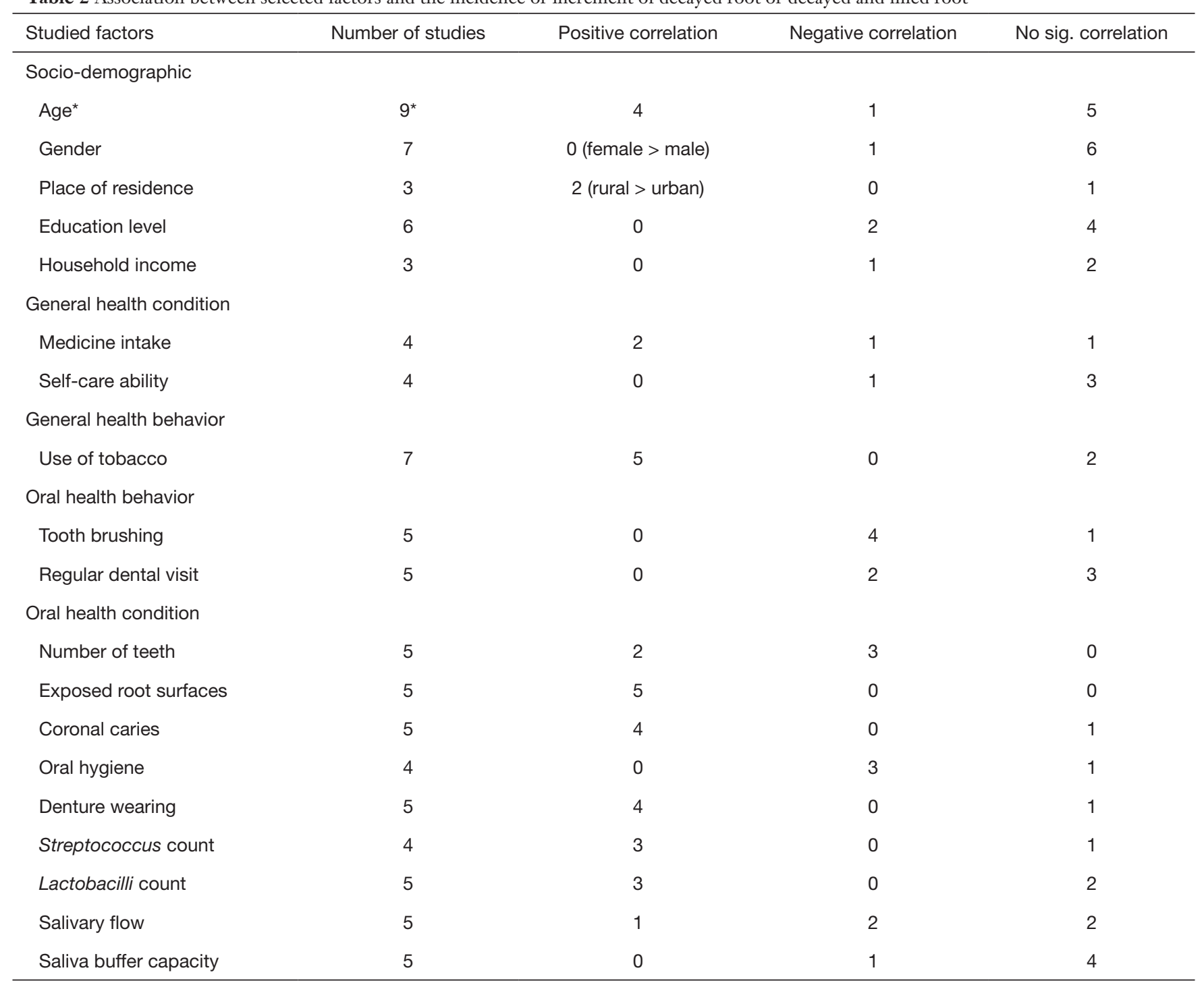

*, in the factor "age", two directions of correlation were reported in one study (Gilbert et al. 2001), in which there was a positive correlation with incidence of DF-root and a negative correlation with incidence of D-root.

sectional studies found that people who lived in the capital city or in urban areas had a lower prevalence of or less root caries than people who lived in towns or rural areas (Table 1). Two out of the 3 included cohort studies found the same situation (Table 2). Thus, it seems that root caries is a more serious problem among the adults living in rural areas.

\section{Education level}

It is noteworthy that two thirds of the cross-sectional studies (8 out of 12) and longitudinal studies (4 out of 6 ) did not find a statistically significant correlation between education level and root caries. The other third of the studies reported a higher prevalence or incidence of root caries among people with lower educational level. Thus, despite the education level of the adult population raises gradually in most countries around the world in recent years, one cannot expect that the root caries situation among the older adults will improve significantly because of this.

\section{Housebold income}

Most (9 out of 11) of the cross-sectional studies found that household income had a negative correlation with root 
caries (Table 1). This suggests that the lower income group is a higher priority group needing more attention when planning dental care services for managing root caries. However, among the three included cohort studies, two did not find a significant relationship (Table 2). So, there is a lack of evidence to support using low income as a predictor of new root caries.

Although the relationships between root caries and socio-economic factors are presented individually in the above section, it should be noted that these factors are inter-related. For instance, people in higher socioeconomic position usually have higher education level and higher income, and live in urban areas. Previous studies had reported more dental caries among the lower socio-economic classes (11). People with lower education or income also have poorer oral health knowledge and behaviors $(11,12)$. Besides, people living in rural areas usually have difficulties accessing proper dental care (13). These are probably important risk factors of root caries and there is a need to provide more oral health education and dental caries prevention services to the disadvantaged population groups.

\section{General health condition}

In the reviewed studies, comorbidity as reflected by the number of medicine/drugs a person took was used as a proxy of the overall systemic health status. The findings from the cross-sectional and longitudinal studies on the relationship between the number of medicines taken and root caries were inconsistent, as similar number of studies (two or three in each category) showed a positive, a negative, and no significant correlation. Most (3 out of 4) of the cross-sectional studies found that adults with lower self-care ability had more root caries (Table 1). It should be noted that self-care ability and general health conditions usually decreases with age. Because there is a positive association between age and DF-root, it is logical that lower self-care ability and poorer general health condition are associated with higher root caries experience. Despite this, most (3 out of 4 ) of the included longitudinal studies did not find a significant correlation between self-care ability and root caries incidence. Furthermore, there were no significant correlations between the prevalence or incidence of root caries, and systemic diseases, depression and body mass index. In summary, results of the systematic reviews do not provide clear evidence to draw a relationship between general health and root caries.

\section{General health behavior}

\section{Use of tobacco}

In 5 out of the 10 included cross-sectional studies, tobacco users had more root caries (Table 1). Consistent with the findings of the surveys, in five of the seven cohort studies, use of tobacco was positively correlated with development of new DF-root or D-root (Table 2). These findings are consistent with those of earlier reviews $(14,15)$. The above findings may be partly explained by the fact that smokers usually have more dental plaque, advanced periodontal diseases and exposed root surfaces (16). Thus, helping older adults to quit smoking may have a preventive effect on root caries besides the benefits on systemic health. More collaboration between the oral and general health care workers would be needed in health promotion.

\section{Alcobol consumption}

Regarding the association between root caries and alcohol consumption, a positive correlation was reported in a crosssectional study (17) while the other three included surveys did not find a significant relationship (Table 1). There was no cohort study reporting on this behavior. Thus, there is not sufficient evidence for drawing a conclusion on this aspect.

\section{Sugar intake}

Considering sugar intake, half (4 out of 8 ) of the reviewed surveys detected a positive correlation with root caries, while the other studies did not (Table 1). One cohort study reported a negative correlative between increment of DFroot and the frequency of sugar consumption (18). These findings are in line with those on the relationship between dietary sugars and dental caries (19), though the strength of evidence for root caries is weaker.

\section{Oral health behavior}

\section{Tooth brushing}

Among the 11 cross-sectional studies that investigated frequency of tooth brushing and root caries, four reported a negative correlation while seven did not (Table 1). On the contrary, most (4 out of 5) of the longitudinal studies on this factor found a negative correlation with new root caries (Table 2). Furthermore, two cohort studies found that people who regularly used mouthwash had lower new root caries. 
Thus, improving people's oral hygiene practices should be considered as an important component of the root caries preventive measures.

\section{Dental visit}

The reported relationship between dental attendance and root caries was inconsistent. One cross-sectional study found people who visited dentists regularly had a higher prevalence of DF-root while three surveys reported the opposite (Table 1). The relationship found in most (3 out of 5) of the included cohort studies was not statistically significant (Table 2). The contrasting findings may be explained by the differences in the reasons for having a dental visit. Most of the reviewed papers did not report if the dental visits of the study participants were for regular check-up or for dental treatment. For people who visit a dentist due to dental problems, they probably have more root caries than those who visit a dentist regularly for checkup. Provision of topical fluorides and other prevention to the latter group will also reduce their root caries risk (20).

\section{Oral health condition}

\section{Clinical conditions}

There was no consistent findings on the number of teeth and root caries as 8 out of the 13 cross-sectional studies reported a negative correlation while four studies reported a positive correlation (Table 1). Similarly, in the reviewed longitudinal studies, the reported relationship between new root caries and the number of teeth was inconsistent since among the five studies, two reported a positive while three reported a negative correlation (Table 2). The relationship found in this recent review is not as clear as that reported in an earlier review (21). This may be due to the different ways used in measuring the number of teeth in the different studies, e.g., some used a continuous scale while others put the number of teeth into different categories.

In contrast, the relationship between root caries and exposure of tooth root surface is much clearer. Among the eight included cross-sectional studies, six reported having more exposed root surfaces due to gingival recession increased the risk of having root caries (Table 1). Furthermore, the amount of gingival recession was reported to have a positive correlation with root caries in all of the three included surveys. The above findings were also reported in longitudinal studies. In all of the five cohort studies, a positive correlation between development of new root caries and the number of root surfaces with recession or amount of gingival recession at baseline was found (Table 2). The above findings are expected because root caries only develops on exposed root surfaces covered by plaque and thus the risk of having new root caries would naturally increase with the amount of exposed tooth root surfaces. This may also partly explain why in this review older age was found to be a predictor of root caries since older adults usually have more gingival recession (22). Prevention of gingival recession, e.g., through prevention of periodontal diseases and correction of traumatic oral hygiene practices, would contribute significantly to prevention of root caries.

All six included cross-sectional studies found a positive correlation between coronal caries and root caries (Table 1). In nearly all of the included cohort studies, positive correlations between new root caries and baseline coronal caries (4 out of 5) and root caries experience (9 out of 9) were also found (Table 2). This finding is consistent with that of an earlier review that past caries experience is a good predictor of root caries (21). Thus, in clinical practice, more intensive root caries prevention such as more frequent topical fluoride applications should be provided to patients who have decayed or filled teeth.

Regarding the influence of oral hygiene on root caries, the vast majority (9 out of 10) of the cross-sectional studies reported that people with less dental plaque had less root caries (Table 1). Similarly, among the four cohort studies, three found a positive correlation between the amount of plaque and new root caries (Table 2). This correlation is expected because root caries is caused by the plaque bacteria on the tooth root surface. Thus, keeping good oral hygiene and not allowing plaque to accumulate on exposed root surfaces is of paramount importance in preventing root caries.

Regarding denture wearing, most (6 out of 10) of the included cross-sectional studies found that denture wearers had more root caries (Table 1). Majority (4 out of 5) of the longitudinal studies also reported a positive correlation. This is probably because denture is a plaque retentive factor in the mouth. Thus, caries preventive measures should be applied regularly to denture wearers and particularly to the exposed tooth roots close to a denture.

\section{Oral microbiota}

In most of the reviewed cross-sectional studies, higher counts of Streptococcus mutans (3 out of the 5 surveys) and Lactobacilli sp. (5 out of the 7 surveys) were associated 
with more root caries experience (Table 1). Similar findings were reported in longitudinal studies (Table 2). Development of new root caries was positively correlated with the Streptococcus mutans count in most (3 out of 4) of the included cohort studies and with the amount of Lactobacilli sp. in 3 of the 5 studies. Furthermore, another cohort study reported a positive correlation between new DF-root and the co-existence of the above two bacterial species (23). Cohort studies also found more new DF-root when there was presence of Streptococcus sobrinus (24) and when there was more Candida (25). Besides, presence of Prevotella was negatively correlated with the incidence of DF-root in the adjusted data analysis of another study (26). It has been reported in earlier reviews on dental caries that poor oral hygiene and presence of Streptococcus mutans and Lactobacilli are associated with caries risk $(21,27)$, and the present reviews found that these factors also increased the prevalence and incidence of root caries.

Although relationships between presence of some species of cariogenic bacteria and root caries have been found, it should be noted that these may not be cause-effect relationships. To have a better understanding of the complex relationship between them, experimental laboratory studies and clinical trials need to be conducted.

\section{Saliva}

A positive correlation between root caries and xerostomia was reported in four of the included cross-sectional studies while three studies did not (Table 1). Among the five included surveys, only one and three of them found higher salivary flow rate and saliva $\mathrm{pH}$ to be negatively correlated with root caries, respectively. Similarly, inconsistent findings were reported in the longitudinal studies (Table 2). Two of the four cohort studies found a higher risk of having new root caries among the participants with a lower salivary flow rate $(18,28)$ while one study reported the opposite $(29)$. Only one of the five cohort studies found a negative correlation between the buffering capacity of saliva and the incidence of DF-root (25) while the other four studies did not find a significant relationship. Although saliva in the mouth is an important environmental factor for dental caries, clear relationships between salivary flow and buffer capacity, and root caries were not found in the two recent systematic review. A possible reason is that these reviews excluded epidemiological studies conducted on populations with special health conditions including those with salivary gland diseases.

\section{Summary}

In the recent review of cross-sectional epidemiological studies, associations between root caries and various factors were found. It is concluded that older adults, smokers, people with lower socio-economic status, those with poorer oral hygiene and those with more exposed root surfaces are at higher risk of having root caries. The identification of risk factors helps the oral health care professionals and public health workers to identify population groups that should have higher priority for receiving root caries preventive measures. More oral health education regarding prevention of root caries should also be provided to these people. The government and dental public health workers should make good use of the information when designing community based preventive strategy for root caries, especially for the aging populations.

In the recent systematic review on cohort studies, the above-mentioned risk factors were also found to be predictors of new root caries. These factors can be used in root caries risk assessment. The information are also valuable for building root caries prediction models. Through the use of root caries assessment and prediction models, people who are more likely to develop root caries can be identified and more preventive measures can be provided. Appropriate root caries prevention and effective management strategies can be adopted according to the individual and population needs. The key factors would include prevention of gingival recession, fluoride application and promotion of good plaque control, especially on exposed tooth root surfaces.

\section{Acknowledgments}

We would like to thank Dr. Devish Sardana, Dr. Katherine Chiu Man Leung and Prof. May Chun Mei Wong for their contributions in conducting the two systematic reviews. Funding: None.

\section{Footnote}

Conflicts of Interest: Both authors have completed the ICMJE uniform disclosure form (available at https://fomm. amegroups.com/article/view/10.21037/fomm.2020.03.02/ coif). The authors have no conflicts of interest to declare.

Ethical Statement: The authors are accountable for all 
aspects of the work in ensuring that questions related to the accuracy or integrity of any part of the work are appropriately investigated and resolved.

Open Access Statement: This is an Open Access article distributed in accordance with the Creative Commons Attribution-NonCommercial-NoDerivs 4.0 International License (CC BY-NC-ND 4.0), which permits the noncommercial replication and distribution of the article with the strict proviso that no changes or edits are made and the original work is properly cited (including links to both the formal publication through the relevant DOI and the license). See: https://creativecommons.org/licenses/by-nc-nd/4.0/.

\section{References}

1. United Nations Department of Economic and Social Affairs. World population ageing 2019 highlights. United Nations, New York, 2019. [Accessed 12 November 2019]. Available online: https://www.un.org/en/ development/desa/population/publications/pdf/ageing/ WorldPopulationAgeing2019-Highlights.pdf

2. Kassebaum NJ, Bernabé E, Dahiya M, et al. Global burden of untreated caries: a systematic review and metaregression. J Dent Res 2015;94:650-8.

3. Gao YB, Hu T, Zhou X, et al. How root caries differs between middle-aged people and the elderly: findings from the 4th national oral health survey of China. Chin J Dent Res 2018;21:221-9.

4. Cronin M, Meaney S, Jepson NJ, et al. A qualitative study of trends in patient preferences for the management of the partially dentate state, Gerodontology 2009;26:137-42.

5. Banting DW. The diagnosis of root caries. J Dent Educ 2001;65:991-6.

6. Bignozzi I, Crea A, Capri D, et al. Root caries: a periodontal perspective. J Periodontal Res 2014;49:143-63.

7. Zhang J, Sardana D, Wong MCM, et al. Factors associated with dental root caries: a systematic review. JDR Clin Trans Res 2020;5:13-29.

8. Zhang J, Leung KCM, Sardana D, et al. Risk predictors of dental root caries: a systematic review. J Dent 2019;89:103166.

9. Wells G, Shea B, O'connell D, et al. The Newcastle-Ottawa Scale for assessing the quality of studies in meta-analyses. Ottawa, Canada: Ottawa Hospital Research Institute, 2016.

10. Gilbert GH, Duncan RP, Dolan TA, et al. Twenty-four month incidence of root caries among a diverse group of adults. Caries Res 2001;35:366-75.
11. Costa SM, Martins CC, Bonfim M, et al. A systematic review of socioeconomic indicators and dental caries in adults. Int J Environ Res Public Health 2012;9:3540-74.

12. Listl S. Income-related inequalities in dental service utilization by Europeans aged 50+. J Dent Res 2011;90:717-23.

13. Brennan D, Spencer AJ, Szuster F. Rates of dental service provision between capital city and noncapital locations in Australian private general practice. Aust J Rural Health 1998;6:12-7.

14. Reibel J. Tobacco and oral diseases. Med Princ Pract 2003;12:22-32.

15. Winn DM. Tobacco use and oral disease. J Dent Educ 2001;65:306-12.

16. Leite FRM, Nascimento GG, Scheutz F, et al. Effect of Smoking on Periodontitis: A Systematic Review and Metaregression. Am J Prev Med 2018;54:831-41.

17. Du M, Jiang H, Tai B, et al. Root caries patterns and risk factors of middle-aged and elderly people in China. Community Dent Oral Epidemiol 2009;37:260-6.

18. Fure S. Ten-year cross-sectional and incidence study of coronal and root caries and some related factors in elderly Swedish individuals. Gerodontology 2004;21:130-40.

19. Sheiham A, James WPT. Diet and dental caries: the pivotal role of free sugars reemphasized. J Dent Res 2015;94:1341-7.

20. Wierichs RJ, Meyer-Lueckel H. Systematic review on noninvasive treatment of root caries lesions. J Dent Res 2015;94:261-71.

21. Ritter AV, Shugars DA, Bader JD. Root caries risk indicators: a systematic review of risk models. Community Dent Oral Epidemiol 2010;38:383-97.

22. Kassab MM, Cohen RE. The etiology and prevalence of gingival recession. J Am Dent. Assoc 2003;134:220-5.

23. Powell LV, Leroux BG, Persson RE, et al. Factors associated with caries incidence in an elderly population, Community Dent Oral Epidemiol 1998;26:170-6.

24. Fure $S$. Five-year incidence of caries, salivary and microbial conditions in 60-, 70-and 80-year-old Swedish individuals. Caries Res 1998;32:166-74.

25. Scheinin A, Pienihäkkinen K, Tiekso J, et al. Multifactorial modeling for root caries prediction: 3-year follow-up results. Community Dent Oral Epidemiol 1994;22:126-9.

26. Lawrence HP, Hunt RJ, Beck JD. Three-year root caries incidence and risk modeling in older adults in North Carolina. J Public Health Dent 1995;55:69-78.

27. Gati D, Vieira AR. Elderly at greater risk for root caries: a look at the multifactorial risks with emphasis on genetics susceptibility. Int J Dent 2011;2011:647168. 
28. Bidinotto AB, Martins AB, dos Santos CM, et al. Fouryear incidence rate and predictors of root caries among community-dwelling south Brazilian older adults, Community Dent. Oral Epidemiol 2018;46:125-31.

doi: 10.21037/fomm.2020.03.02

Cite this article as: Zhang J, Lo EC. Epidemiology of dental root caries: a review of risk factors. Front Oral Maxillofac Med 2020;2:5.
29. Hayes M, Da Mata C, Cole M, et al. Risk indicators associated with root caries in independently living older adults. J Dent 2016;51:8-14. 